\title{
Urinary bacterial profile and antibiotic susceptibility pattern among pregnant women in Rahima Moosa Mother and Child Hospital, Johannesburg
}

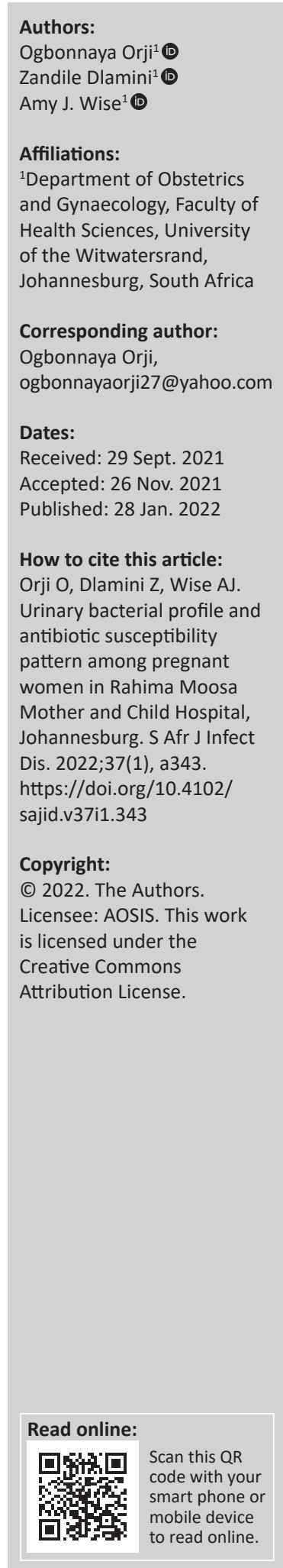

\begin{abstract}
Background: Urinary tract infection (UTI) in pregnancy is associated with significant morbidity for both the mother and the foetus. The aim of this study was to determine the prevalence of UTI, urinary bacterial susceptibility, and resistance patterns among pregnant women with a possible UTI at Rahima Moosa Mother and Child Hospital (RMMCH) in Johannesburg.
\end{abstract}

Methods: In this retrospective study, we analysed mid-stream urine culture and antibiotic susceptibility data from both inpatients and outpatients of pregnant women who attended RMMCH from January 2017 to December 2017. Data were collected from patients' files and then matched with urine microscopy, sensitivity and culture (MC\&S) results from the National Health Laboratory Services (NHLS) data.

Results: Urine microscopy, cultures and sensitivities were performed on 1984 specimens belonging to pregnant women who presented with symptoms and/or signs of a UTI. A total of 333 patients (16.8\%) had positive bacterial cultures. Escherichia coli (E. coli) was the commonest bacterial isolate (49.9\%). Other microorganisms isolated included Klebsiella species (14.4\%), Enterococcus faecalis (12.9\%) and coagulase-negative staphylococci (CoNS); $(8.9 \%)$. Approximately $98 \%$ of organisms were sensitive to cephalexin. Cefuroxime $(95.2 \%)$, ceftriaxone/cefotaxime $(94.4 \%)$ and nitrofurantoin $(81.9 \%)$ demonstrated antimicrobial effectiveness as indicated. Most isolates were resistant to ampicillin/amoxicillin (84.4\%), Trimethoprim/Sulfamethoxazole (55.6\%) and amoxicillin-clavulanic acid (50.2\%).

Conclusion: E. coli was the commonest pathogen causing UTIs in pregnancy with Enterococcus faecalis increasing in prevalence. The choice of antimicrobial therapy in pregnancy should be determined according to sensitivity and resistance and foeto-maternal safety.

Keywords: UTI; sensitivity; urine microscopy; sensitivity and culture; pathogen; pregnancy.

\section{Introduction}

Several changes occur during pregnancy that predispose pregnant women to urinary tract infections (UTIs). Physiological, physical, mechanical and hormonal changes result in increased urinary stasis. Altered urine composition with elevated glucose levels coupled with a short urethra ( $3 \mathrm{~cm}-4 \mathrm{~cm}$ in women) increases the predisposition to UTIs in pregnant women. ${ }^{1}$ The prevalence of UTI in pregnancy ranges between $2 \%$ and $10 \%$ globally. $2,6,7,8$

Urinary tract infections are among the commonest bacterial infections complicating pregnancy. 2,3,4 Urinary tract infection can be either symptomatic or asymptomatic. A symptomatic UTI patient is one with significant bacteriuria and with symptoms of a UTI. Whereas, a condition characterised by lack of symptoms of UTI with significant bacterial yielding positive urine cultures $\left(\geq 10^{5}\right.$ colony forming units / millilitre [CFU/mL]) is called an asymptomatic UTI (asymptomatic bacteriuria). ${ }^{5}$

However, symptoms like dysuria and frequency are common in pregnancy, but have a very low specificity for a true UTI. Other symptoms such as urethritis, cystitis or pyelonephritis may be present. Clinicians can be wrong in their suspicion of a UTI from the symptoms alone. Many of those with symptoms and positive cultures may therefore have had asymptomatic bacteriuria instead. ${ }^{6}$ The significance of UTI in pregnancy, in view of its associated maternal and foetal morbidity and mortality, has been widely evaluated.

The occurrence of UTI in pregnancy is increased by several factors. The highest incidence has been reported in African-American multiparous women, while the lowest incidence occurs among affluent white women of low parity. ${ }^{6}$ Poor socio-economic status is a significant risk factor, with 
indigent women having a five-fold increased risk of acquiring UTIs. ${ }^{9}$ Other risk factors include: increasing maternal age, high parity, reduced immune function, poor perineal hygiene, a history of recurrent UTI, diabetes mellitus, neurogenic urinary retention, anatomic or functional urinary tract abnormalities, and increased frequency of sexual activity. ${ }^{2,10,11}$

Studies in developing countries show that UTIs are usually present at the first antenatal visit and less than $1 \%$ of women develop bacteriuria after a negative screen in early pregnancy. ${ }^{2}$ A UTI in pregnancy contributes to significant maternal and perinatal morbidity and mortality. Maternal complications include overt pyelonephritis in $25 \%-40 \%$ of previously asymptomatic women as the pregnancy progresses, and in $1 \%-2 \%$ in those with symptomatic infections., ${ }^{2,12}$ Other maternal complications include: anaemia, miscarriages, preterm labour, hypertension, pre-eclampsia, puerperal sepsis, chronic pyelonephritis and occasionally, renal failure..$^{2,8,13}$ Urinary tract infections are also associated with foetal growth restriction, prematurity, low birthweight and foetal death. ${ }^{13,14}$

The causative organisms arise from the normal vaginal, perineal and faecal flora. ${ }^{3,13}$ These include: Escherichia coli (E. coli), Staphylococcus aureus, Enterococcus faecalis, Proteus mirabilis, Klebsiella species, and Streptococcus species, among others. ${ }^{2}$ There are numerous reports of resistance to antimicrobials by urinary tract pathogens. ${ }^{14,15}$ Antimicrobial resistance in these organisms occurs because of broad-spectrum antibiotic abuse in humans and in animal feeds. ${ }^{13,14}$

Antibiotic resistance is frequently observed in nosocomial settings. However, it is also becoming apparent in communityacquired UTIs, with an increasing incidence of Gram-positive cocci, for example, Staphylococci sp. and Gram-negative organisms such as Klebsiella sp. becoming more prevalent. ${ }^{2,16,17}$

Urinary tract infections may present as acute infections and the administration of antibiotics may be necessary while awaiting microscopy, sensitivity and culture (MC\&S) results to prevent and/or reduce maternal and foetal morbidity and mortality especially in low-resourced countries.

The aim of this study was to determine the antibiotic sensitivity pattern among pregnant women with symptomatic UTIs and to describe the pathogenicity and antibiotic susceptibility among the causative bacterial organisms. ${ }^{18}$

Knowledge of the local bacterial and susceptibility patterns can guide the judicious use of empiric therapy. ${ }^{19}$

\section{Methods}

\section{Setting and study design}

The study was conducted at the Rahima Moosa Mother and Child Hospital (RMMCH), which is a regional hospital. The hospital serves a population of approximately 200000 women and children in three regions in the Gauteng province. ${ }^{20} \mathrm{An}$ average of 1700 women attend the antenatal clinic on a monthly basis, including high- and low-risk women.

In this retrospective study, we analysed mid-stream urine culture and antibiotic susceptibility data from both inpatients and outpatients of pregnant women who attended RMMCH from January 2017 to December 2017. The National Health Laboratory Services (NHLS) was approached for a list of all samples sent in the specified time period. All available files from those that were culture positive were retrieved and reviewed. The NHLS data uses a pre-defined procedure for culturing, bacterial identification, and susceptibility testing.

\section{Urine sample collection, primary inoculation, and analysis}

The samples were collected during routine clinical care, at the discretion of the treating clinician, and it is not possible to comment on the correctness of the technique or time taken to reach the laboratory. The reason for sending a sample was not always reflected in the available notes.

Urine samples were cultured on 5\% blood agar and MacConkey agar using calibrated loops in a semi-quantitative assessment and incubated in aerobic conditions at $35^{\circ} \mathrm{C}-37^{\circ} \mathrm{C}$ for $18 \mathrm{~h}-24$ h. $^{2}$ Isolates were identified and confirmed using standard methods including Gram staining; colony morphology on media; growth on selective media; lactose and mannitol fermentation; hydrogen sulphide production; catalase, oxidase, coagulase, and indole tests; citrate utilisation; and urease testing. Urine infection cultures were considered positive with bacterial counts $\geq 10^{5} / \mathrm{mL}^{2,21}$ All patients with positive urine cultures were treated. ${ }^{2}$

\section{Antimicrobial susceptibility testing}

For reliable detection, laboratories may use conventional, quantitative susceptibility testing methods or specially developed, single concentration agar screening tests for some resistant species. ${ }^{2}$

Antimicrobial susceptibility testing for UTIs in the laboratory is performed using two groups of antimicrobial discs for cascade reporting, a strategy recommended by the Clinical and Laboratory Standards Institute. In this strategy, the reporting of antimicrobial susceptibility test results for the second group of agents (e.g. broader- spectrum, more costly) may only be reported if an organism is resistant to primary agents within a particular drug class. If a pathogen shows resistance to all of these, the laboratory will move to the second stage for testing broad-spectrum antibiotics. ${ }^{2}$

Identification and sensitivity testing were done if the culture was pure and growth was significant $\left(\geq 10^{5} \mathrm{CFU} / \mathrm{mL}\right)$. If the culture growth involved a mix of two pathogens and no isolate was dominant, or more than two types of colonies were grown, then it was reported as a mixed growth (contaminants) and clinical correlation was needed to make a determination. In such cases, no sensitivity testing was carried out.,22 


\section{Statistical analysis}

All the data collected were managed using Research Electronic Data Capture (REDCap) electronic data capture tools hosted at a University in Johannesburg. The REDCap is a secure, web-based application designed to support data capture for research studies. ${ }^{23,24}$ The biostatisticians at the University of the Witwatersrand (WITS) in Johannesburg assisted in the analysis phase of the study. The data were analysed using Stata ${ }^{\circledR}$ version 13.0.15. ${ }^{25}$ Descriptive data was expressed using means with ranges and medians with standard deviations (s.d.).

\section{Ethical considerations}

Ethical clearance was obtained from the Human Research Ethics Committee (Medical) of the University of the Witwatersrand, reference number: M181070.

\section{Results}

A total of 1984 urine samples from pregnant women with suspected UTI were selected for isolation and identification of bacteria and antimicrobial susceptibility testing in both inpatients and outpatients at our centre, 333 urine samples $(16.8 \%)$ were culture positive for UTI, while 1599 urine samples $(80.6 \%)$ were culture negative and 52 urine samples $(2.6 \%)$ were identified as contaminants and contaminated urine were determined by more than five epithelial cells on microscopic evaluation, specific gravidity $>1.035$ and urine culture with polymicrobial growth - that is, urine culture that contain more than one organisms (Figure 1).

In all, 324 women (97.3\%) were booked for antenatal care. The ages of the study cohort ranged between 16 and 44 years, with the majority (58.9\%) in the 20 and 29-year age group. There were 52 (15.6\%) human immunodeficiency virus (HIV) infected women. The highest frequency of UTIs observed ( $n=229,68.8 \%)$ were from specimens taken in the third trimester. Gestational ages ranged between 26 weeks and 40 weeks and birthweights from $619 \mathrm{~g}$ to $4495 \mathrm{~g}$. Of the 333 patients, $29.1 \%(97 / 333)$ were inpatients and $70.9 \%(236 / 333)$ were outpatients (Table 1 ).

The most frequently identified microorganism was E. coli (49.9\%), followed by Klebsiella (14.4\%), Enterococci faecalis (12.9\%) and coagulase-negative Staphylococci (CoNS) (8.9\%) (Table 2).

Table 3 shows that microbes demonstrated high susceptibility to cephalosporin: cephalexin $(98.0 \%)$, cefuroxime $(95.1 \%)$ and ceftriaxone/cefotaxime (94.4\%), while $81.9 \%$ of the isolates were sensitive to nitrofurantoin. Piperacillin/ tazobactam and gentamicin had an overall susceptibility of $96.2 \%$ and $70.1 \%$ respectively. The resistance observed in this study was gentamicin $(29.7 \%)$, nitrofurantoin $(13.5 \%)$ and piperacillin/tazobactam $(2.0 \%)$, while intermediate resistance was $0.2 \%$ for gentamicin, $1.8 \%$ for piperacillin/tazobactam and $4.6 \%$ for nitrofurantoin.

Amikacin, chloramphenicol, imipenem, linezolid, tobramycin, ertapenem, vancomycin and colistin sulphate had the highest

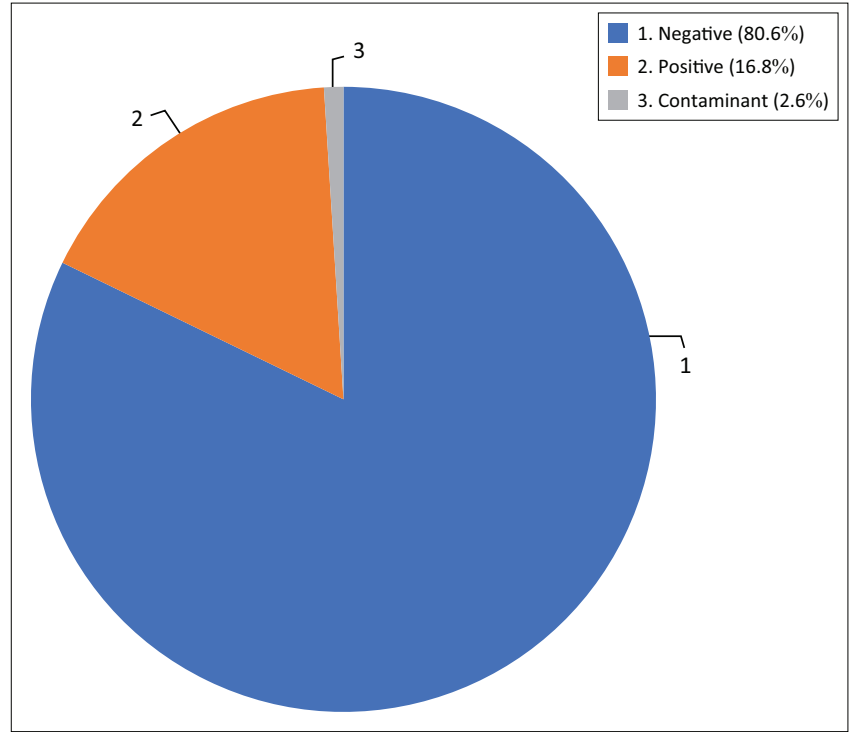

FIGURE 1: Culture results of urine specimens submitted for microscopy, sensitivity and culture.

TABLE 1: Demographic, antenatal and treatment data of women with positive microscopy, sensitivity and cultures.

\begin{tabular}{|c|c|c|}
\hline Variable & $\begin{array}{l}\text { Number } \\
N=333\end{array}$ & Percentages (\%) \\
\hline \multicolumn{3}{|l|}{ Age (years) } \\
\hline$<20$ & 39 & 11.7 \\
\hline $20-29$ & 170 & 51.1 \\
\hline 30-39 & 110 & 33.0 \\
\hline$>40$ & 14 & 4.2 \\
\hline \multicolumn{3}{|l|}{ Parity } \\
\hline 0 & 132 & 39.7 \\
\hline 1 & 68 & 20.4 \\
\hline 2 & 81 & 24.3 \\
\hline 3 & 36 & 10.8 \\
\hline 4 & 15 & 4.5 \\
\hline 5 & 1 & 0.3 \\
\hline \multicolumn{3}{|l|}{ Gravidity } \\
\hline 1 & 106 & 31.9 \\
\hline 2 & 72 & 21.6 \\
\hline 3 & 75 & 22.5 \\
\hline 4 & 45 & 13.5 \\
\hline 5 & 18 & 5.4 \\
\hline 6 & 16 & 4.8 \\
\hline 7 & 1 & 0.3 \\
\hline \multicolumn{3}{|c|}{ Trimester during which urine MC\&S was done } \\
\hline First trimester & 2 & 0.6 \\
\hline Second trimester & 102 & 30.6 \\
\hline Third trimester & 229 & 68.8 \\
\hline \multicolumn{3}{|c|}{ ANC attendance (at least once) } \\
\hline Booked & 324 & 97.3 \\
\hline Unbooked & 9 & 2.7 \\
\hline \multicolumn{3}{|l|}{ HIV } \\
\hline Negative & 281 & 84.4 \\
\hline Positive & 52 & 15.6 \\
\hline Recurrent infection & 5 & 1.50 \\
\hline \multicolumn{3}{|l|}{ Admission status } \\
\hline Inpatient & 97 & 29.1 \\
\hline Outpatient & 236 & 70.9 \\
\hline
\end{tabular}

MC\&S, microscopy, sensitivity and culture; ANC, antenatal care; HIV, human immunodeficiency virus. 
overall potency of $100 \%$ each to all isolated microbials tested against them. Co-amoxiclav, cotrimoxazole, ampicillin/ amoxicillin, clindamycin, nalidixic acid and erythromycin/ azithromycin were less than $50 \%$ effective against the cultured microbes.

Table 4 shows antimicrobial sensitivity to gram-negative microbials. Regarding E. coli, most cultures were $100 \%$ sensitive to amikacin, imipenem, ertapenem, meropenem. However, the majority were resistant to nalidixic acid, ampicillin/amoxicillin and co-amoxiclav.

All the Klebsiella were sensitive to amikacin, ertapenem and imipenem, while nearly half of Klebsiella isolates were sensitive to different cephalosporins whereas only $20.9 \%$

TABLE 2: Number and percentage of microorganisms isolated from pregnant women urine samples in the study.

\begin{tabular}{lcc}
\hline Microorganism & Number $(\boldsymbol{N})$ & Percentage (\%) \\
\hline Escherichia coli & 166 & 49.9 \\
Klebsiella species & 48 & 14.4 \\
Enterococcus faecalis (Group D streptococci) & 43 & 12.9 \\
Coagulase- negative staphylococci (CoNS) & 30 & 8.9 \\
Proteus & 3 & 0.9 \\
Enterobacter species & 3 & 0.9 \\
Streptococcus agalactiae (Group B) & 15 & 4.5 \\
Staphylococcus spp, (other non-CoNS or & 8 & 2.4 \\
S. aureus) & & \\
Staphylococcus aureus & 8 & 2.4 \\
Acinetobacter & 2 & 0.7 \\
Other Microorganisms (Corynebacterium, & 7 & 2.1 \\
Pseudomonas) & & \\
\hline
\end{tabular}

CoNS, coagulase-negative staphylococci. were sensitive to nitrofurantoin. Enterobacter species showed $100 \%$ sensitivity to amikacin, ciprofloxacin, co-trimoxazole, ertapenem and imipenem. These cultures were most resistant to co-amoxiclav and cefuroxime. The three Proteus species were sensitive to most tested antibiotics except ceftriaxone, with a $75 \%$ reported resistance.

Acinetobacter species was cultured twice and showed resistance to ampicillin/amoxicillin, co-amoxiclav, cefuroxime, nalidixic acid and nitrofurantoin. Both were sensitive to the carbapenems.

All Enterococcus faecalis culture were sensitive to ampicillin/amoxicillin, co-amoxiclav, nitrofurantoin and vancomycin, but were all resistant to co-trimoxazole, gentamicin, cefuroxime and nalidixic acid while CoNS isolates were sensitive to tigecycline, vancomycin, ampicillin/amoxicillin, co-amoxiclav and gentamicin. (Table 5). Most streptococcus agalactiae (Group B) isolates were sensitive to co-amoxiclav, cefuroxime, vancomycin, ampicillin/amoxicillin and nitrofurantoin (Table 5).

\section{Discussion}

Pregnant women are at increased risk of developing UTI, mainly because of a shift in the position of the urinary tract and hormonal changes that occur throughout pregnancy, thus making it easier for bacteria to reach the kidney and leading to both symptomatic and asymptomatic bacteriuria. $2,26,27,28$

TABLE 3: Overall sensitivity of antimicrobial agents.

\begin{tabular}{|c|c|c|c|c|c|c|c|}
\hline \multirow[t]{2}{*}{ Antimicrobial agents } & \multicolumn{2}{|c|}{ Sensitive } & \multicolumn{2}{|c|}{ Resistant } & \multicolumn{2}{|c|}{ Intermediate } & \multirow[t]{2}{*}{ Total } \\
\hline & $N$ & $\%$ & $N$ & $\%$ & $N$ & $\%$ & \\
\hline Amikacin $\dagger$ & 198 & 100 & - & - & - & - & 198 \\
\hline Ampicillin/Amoxicillin & 48 & 15.0 & 273 & 84.4 & 2 & 0.6 & 324 \\
\hline Amoxicillin + clavulanic acid & 146 & 47.3 & 155 & 50.2 & 8 & 2.5 & 309 \\
\hline Cefotaxime/Ceftriaxone & 291 & 94.4 & 17 & 5.6 & - & - & 308 \\
\hline Ceftazidime & 260 & 92.0 & 22 & 8.0 & - & - & 282 \\
\hline Cephalexin/Cephradine & 323 & 98 & 7 & 2 & - & - & 330 \\
\hline Chloramphenicol & 6 & 100 & - & - & - & - & 6 \\
\hline Ciprofloxacin & 180 & 80.2 & 41 & 18.4 & 3 & 1.4 & 224 \\
\hline Clindamycin & 10 & 40.0 & 13 & 55.5 & 1 & 5.5 & 24 \\
\hline Colistin Sulphate & 2 & 100 & - & - & - & - & 2 \\
\hline Ertapenem $\dagger$ & 118 & 100 & - & - & - & - & 118 \\
\hline Erythromycin/Azithromycin & 8 & 16.7 & 34 & 70.8 & 6 & 12.5 & 48 \\
\hline Imipenem $\dagger$ & 203 & 100 & - & - & - & - & 203 \\
\hline Linezolid & 101 & 100 & - & - & - & - & 101 \\
\hline Meropenem $\dagger$ & 107 & 98.2 & 2 & 1.8 & - & - & 109 \\
\hline Moxifloxacin & 48 & 88.0 & 2 & 4.0 & 4 & 8.0 & 54 \\
\hline Nalidixic acid & 13 & 18.9 & 55 & 81.1 & - & - & 68 \\
\hline Nitrofurantoin & 271 & 81.9 & 45 & 13.5 & 15 & 4.6 & 331 \\
\hline Piperacillin/Tazobactam & 92 & 96.2 & 2 & 2.0 & 2 & 1.8 & 96 \\
\hline Tigecycline $\dagger$ & 69 & 100 & - & - & - & - & 69 \\
\hline Tobramycin & 69 & 84.2 & 8 & 9.3 & 5 & 6.5 & 82 \\
\hline Trimethoprim/Sulfamethoxazole & 129 & 44.4 & 161 & 55.6 & - & - & 290 \\
\hline Vancomycin & 78 & 100 & - & - & - & - & 78 \\
\hline
\end{tabular}

$N$, number, \%, percentages.

$\dagger$, Provided as single discs. 
TABLE 4: Antimicrobial sensitivity in gram-negative bacterial isolates.

\begin{tabular}{|c|c|c|c|c|c|c|c|c|c|c|c|c|c|c|c|}
\hline \multirow{2}{*}{$\begin{array}{l}\text { Gram-negative } \\
\text { bacteria/Antimicrobial }\end{array}$} & \multicolumn{3}{|c|}{ Escherichia coli (166) } & \multicolumn{3}{|c|}{ Klebsiella (48) } & \multicolumn{3}{|c|}{ Enterobacter (3) } & \multicolumn{3}{|c|}{ Proteus (3) } & \multicolumn{3}{|c|}{ Acinetobacter (2) } \\
\hline & S & $\mathbf{R}$ & 1 & S & $\mathbf{R}$ & 1 & $\mathbf{S}$ & $\mathbf{R}$ & 1 & S & $\mathbf{R}$ & 1 & $\mathbf{S}$ & $\mathbf{R}$ & 1 \\
\hline \multicolumn{16}{|l|}{ Amikacin } \\
\hline$N$ & 166 & - & - & 48 & - & - & 3 & - & - & 3 & - & - & - & - & - \\
\hline$\%$ & 100 & - & - & 100 & - & - & 100 & - & - & 100 & - & - & - & - & - \\
\hline \multicolumn{16}{|l|}{ Ampicillin/Amoxicillin } \\
\hline$N$ & 59 & 104 & 3 & - & 43 & - & - & - & - & 2 & - & - & - & 2 & - \\
\hline$\%$ & 35.7 & 62.8 & 1.4 & - & 100 & - & - & - & - & 100 & - & - & - & 100 & - \\
\hline \multicolumn{16}{|l|}{ Co-amoxiclav } \\
\hline$N$ & 80 & 80 & 6 & 27 & 14 & 7 & 1 & 2 & - & 2 & 1 & - & - & 2 & - \\
\hline$\%$ & 48 & 47.6 & 3.4 & 56.1 & 29.3 & 14.6 & 16.7 & 83.3 & - & 75 & 25 & - & - & 100 & - \\
\hline \multicolumn{16}{|l|}{ Cephalexin/Cephradine } \\
\hline$N$ & 106 & 60 & - & 24 & 24 & - & 1 & 2 & - & 3 & - & - & - & 2 & - \\
\hline$\%$ & 63.8 & 36.2 & - & 50 & 50 & - & 25 & 75 & - & 100 & - & - & - & 100 & - \\
\hline \multicolumn{16}{|l|}{ Ceftriaxone } \\
\hline$\%$ & 52.9 & 47.1 & - & 53.7 & 46.7 & - & 75 & 25 & - & 15 & 75 & - & 50 & 50 & - \\
\hline \multicolumn{16}{|l|}{ Cefuroxime } \\
\hline$N$ & 100 & 64 & 2 & 24 & 24 & - & - & - & - & 3 & - & - & - & 2 & - \\
\hline$\%$ & 60.5 & 39 & 0.5 & 50 & 50 & - & - & - & - & 100 & - & - & - & 100 & - \\
\hline \multicolumn{16}{|l|}{ Ciprofloxacin } \\
\hline$N$ & 126 & 40 & - & 47 & 1 & - & 3 & - & - & 3 & - & - & 1 & 1 & - \\
\hline$\%$ & 75.8 & 31.2 & - & 97.3 & 2.7 & - & 100 & - & - & 100 & - & - & 50 & 50 & - \\
\hline Co-trimoxazole & & & & & & & & & & & & & & & \\
\hline$N$ & 55.1 & 43.9 & - & 28 & 20 & - & 3 & - & - & 83.3 & 16.7 & - & 1 & 1 & - \\
\hline$\%$ & - & - & - & 58.1 & 41.9 & - & 100 & - & - & - & - & - & 50 & 50 & - \\
\hline Ertapenem & & & & & & & & & & & & & & & \\
\hline$N$ & 166 & - & - & 28 & - & - & 3 & - & - & 3 & - & - & 2 & - & - \\
\hline$\%$ & 100 & - & - & 100 & - & - & 100 & - & - & 100 & - & - & 100 & - & - \\
\hline Gentamicin & & & & & & & & & & & & & & & \\
\hline Imipenem & & & & & & & & & & & & & & & \\
\hline$N$ & 166 & - & - & 43 & - & - & 3 & - & - & 3 & - & - & 2 & - & - \\
\hline$\%$ & 100 & - & - & 100 & - & - & 100 & - & - & 100 & - & - & 100 & - & - \\
\hline Nalidixic acid & & & & & & & & & & & & & & & \\
\hline$N$ & 63 & 103 & - & 47 & 1 & - & 2 & 1 & - & 2 & 1 & - & - & - & 2 \\
\hline$\%$ & 37.8 & 62.2 & - & 95 & 35 & - & 75 & 25 & - & 75 & 25 & - & - & - & 100 \\
\hline Nitrofurantoin & & & & & & & & & & & & & & & \\
\hline$N$ & 126 & 40 & - & 10 & 22 & 16 & 1 & 2 & - & - & 3 & - & - & - & 2 \\
\hline$\%$ & 75.5 & 25.5 & - & 20.9 & 44.9 & 34.2 & 25 & 75 & - & - & 100 & - & - & - & 100 \\
\hline Tigecycline & & & & & & & & & & & & & & & \\
\hline$N$ & 100 & 66 & - & 36 & 12 & - & 3 & - & - & 3 & - & - & NR & NR & NR \\
\hline$\%$ & 60.0 & 40.0 & - & 75 & 25 & - & 100 & - & - & 100 & - & - & - & - & - \\
\hline Meropenem & & & & & & & & & & & & & & & \\
\hline$N$ & 166 & - & - & 43 & - & - & 2 & - & - & 2 & - & - & 1 & 1 & - \\
\hline$\%$ & 100 & - & - & 100 & - & - & 100 & - & - & 100 & - & - & 50 & 50 & - \\
\hline Piperacillin/Tazobactam & & & & & & & & & & & & & & & \\
\hline$N$ & 159 & 2 & 5 & 40 & - & - & 3 & - & - & 3 & - & - & 1 & 1 & - \\
\hline$\%$ & 95.7 & 1.4 & 2.9 & 100 & - & - & 100 & - & - & 100 & - & - & 50 & 50 & - \\
\hline Tobramycin & & & & & & & & & & & & & & & \\
\hline$N$ & 147 & 13 & 6 & 37 & 11 & - & - & - & - & - & - & - & - & - & - \\
\hline$\%$ & 88.5 & 7.7 & 3.8 & 77.8 & 22.2 & - & - & - & - & - & - & - & - & - & - \\
\hline Colistin sulphate & & & & & & & & & & & & & & & \\
\hline$N$ & NR & NR & NR & NR & - & - & - & - & - & - & - & - & 2 & - & - \\
\hline$\%$ & - & - & - & - & - & - & - & - & - & - & - & - & 100 & - & - \\
\hline
\end{tabular}

$\mathrm{S}$, sensitive; R, resistant; I, intermediate; $\mathrm{NR}$, not recorded; $N$, numbers; \%, percentages.

Symptomatic and asymptomatic bacteriuria are common in pregnant women. We do not perform universal screening with cultures during pregnancy. Thus, asymptomatic bacteriuria during pregnancy which is important may not be detected. This study was conducted only for symptomatic bacteriuria among pregnant women by reviewing their files.

E. coli was the most common gram-negative bacteria isolated in this study, which is in line with the findings of previous 
TABLE 5: Antimicrobial sensitivity in gram-positive bacterial isolates.

\begin{tabular}{|c|c|c|c|c|c|c|c|c|c|c|c|c|c|c|c|c|c|c|}
\hline \multirow[t]{2}{*}{ Gram-positive bacteria } & \multicolumn{3}{|c|}{$\begin{array}{l}\text { Enterococcus } \\
\text { faecalis (43) }\end{array}$} & \multicolumn{3}{|c|}{$\begin{array}{l}\text { Coagulase-negative } \\
\text { staphylococci (30) }\end{array}$} & \multicolumn{3}{|c|}{$\begin{array}{c}\text { Streptococcus } \\
\text { agalactiae } \\
\text { (Group B) (15) }\end{array}$} & \multicolumn{3}{|c|}{$\begin{array}{l}\text { Staphylococcus } \\
\text { aureus (8) }\end{array}$} & \multicolumn{3}{|c|}{$\begin{array}{l}\text { Staphylococcus } \\
\text { spp (8) }\end{array}$} & \multicolumn{3}{|c|}{$\begin{array}{l}\text { Enterococcus } \\
\text { spp (3) }\end{array}$} \\
\hline & $\mathbf{S}$ & $\mathbf{R}$ & 1 & $S$ & $\mathbf{R}$ & 1 & 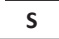 & $\mathbf{R}$ & 1 & $\mathbf{S}$ & $\mathbf{R}$ & 1 & S & $\mathbf{R}$ & 1 & $S$ & $\mathbf{R}$ & 1 \\
\hline \multicolumn{19}{|l|}{ Ampicillin/Amoxicillin } \\
\hline$N$ & 43 & - & - & 26 & 4 & - & 15 & - & - & 3 & 5 & - & 5 & 3 & - & 3 & - & - \\
\hline$\%$ & 100 & - & - & 86.7 & 13.3 & - & 100 & - & - & 46 & 54 & - & 60 & 40 & - & 100 & - & - \\
\hline \multicolumn{19}{|l|}{ Co-amoxiclav } \\
\hline$N$ & 43 & - & - & 26 & 4 & - & 15 & - & - & 3 & 5 & - & 5 & 3 & - & 3 & - & - \\
\hline$\%$ & 100 & - & - & 88.2 & 11.8 & - & 100 & - & - & 49 & 51 & - & 65 & 35 & - & 100 & - & - \\
\hline \multicolumn{19}{|l|}{ Cefepime } \\
\hline$N$ & 24 & 19 & - & 22 & 8 & - & 14 & 1 & - & 2 & 6 & - & 1 & 7 & - & - & - & - \\
\hline$\%$ & 55 & 45 & - & 76.5 & 23.5 & - & 96 & 4 & - & 7 & 83 & - & 10 & 90 & - & - & - & - \\
\hline \multicolumn{19}{|l|}{ Cefuroxime } \\
\hline$N$ & 26 & 17 & - & 24 & 6 & - & 15 & - & - & 3 & 5 & - & 2 & 6 & - & 1 & 2 & - \\
\hline$\%$ & 60 & 40 & - & 81.6 & 18.4 & - & 100 & - & - & 40 & 60 & - & 30 & 70 & - & 25 & 75 & - \\
\hline \multicolumn{19}{|l|}{ Ciprofloxacin } \\
\hline$N$ & 32 & 11 & - & 24 & 4 & 2 & - & - & - & 8 & - & - & - & - & - & 2 & 1 & 1 \\
\hline$\%$ & 75 & 25 & - & 80 & 11.4 & 8.6 & - & - & - & 100 & - & - & - & - & - & 77.8 & 11.1 & - \\
\hline \multicolumn{19}{|l|}{ Cotrimoxazole } \\
\hline$N$ & - & 43 & - & 20 & 10 & - & 11 & 4 & - & 5 & 3 & - & 5 & 3 & - & 1 & 2 & - \\
\hline$\%$ & - & 100 & - & 68.2 & 31.8 & - & 76 & 34 & - & 67 & 33 & - & 65 & 35 & - & 25 & 75 & - \\
\hline \multicolumn{19}{|l|}{ Gentamicin } \\
\hline$N$ & - & 43 & - & 27 & 3 & - & 6 & 9 & - & 3 & 5 & - & 5 & 3 & - & 1 & 2 & - \\
\hline$\%$ & - & 100 & - & 91.4 & 8.6 & - & 42 & 58 & - & 46 & 54 & - & 58 & 42 & - & 4 & 96 & - \\
\hline \multicolumn{19}{|l|}{ Nalidixic } \\
\hline$N$ & - & 43 & - & 1 & 29 & - & 1 & 14 & - & 1 & 7 & - & - & 8 & - & 1 & 2 & - \\
\hline$\%$ & - & 100 & - & 2 & 98 & - & 3 & 97 & - & 3 & 97 & - & - & 100 & - & 1.4 & 98.6 & - \\
\hline \multicolumn{19}{|l|}{ Nitrofurantoin } \\
\hline$N$ & 43 & - & - & 26 & 4 & - & 14 & 1 & - & 7 & 1 & - & 72 & 28 & - & 2 & 1 & - \\
\hline$\%$ & 100 & - & - & 87.9 & 12.1 & - & 95 & 5 & - & 94 & 6 & - & - & - & - & 95 & 5 & - \\
\hline \multicolumn{19}{|l|}{ Vancomycin } \\
\hline$N$ & 43 & - & - & - & - & - & - & - & - & - & - & - & - & - & - & 3 & - & - \\
\hline$\%$ & 100 & - & - & - & - & - & - & - & - & - & - & - & - & - & - & 100 & - & - \\
\hline
\end{tabular}

$\mathrm{S}$, sensitive; $\mathrm{R}$, resistant; I, intermediate; $N$, numbers; $\%$, percentages.

studies, such as that by Tandan et al. and several other studies. ${ }^{1,12,13,15}$ This finding suggests that most organisms causing UTI are from the lower gastrointestinal tract which acts as a reservoir for organisms such as E. coli. ${ }^{1,9}$

Klebsiella species was the next most common gram-negative organism isolated in this study, accounting for $14.4 \%$ of positive cultures, and is similar to the finding in a study conducted in KwaZulu-Natal (20\%). ${ }^{1,29}$

Enterococcus faecalis (12.9\%) was the most frequent grampositive organism detected and had been noted as a significant bacterial isolate from women with UTI in pregnancy in other studies. ${ }^{1,30}$

Empiric therapy should be commenced as soon as urine samples are taken and modified once culture results become available to prevent serious morbidity. ${ }^{1,31}$

Hence, the recommendation of nitrofurantoin as a first line drug for the treatment of UTI in pregnancy. ${ }^{7,12}$ The University's obstetric protocol recommends the use of nitrofurantoin at a dose of $100 \mathrm{mg}$ orally, 6 hourly for 5 days or cefuroxime at a dose of $250 \mathrm{mg}$ orally stat (this is a non-Essential Medicines List (EML) item in SA) ${ }^{32}$ for uncomplicated UTI and twice daily for 5 days as empirical therapy for complicated UTI. ${ }^{33,34,35}$
The use of nitrofurantoin in pregnancy is supported by the most recent American College of Obstetricians and Gynaecologists (ACOG) Committee opinion which concluded that in the second and third trimester it was a suitable choice. In the first trimester it can be used if there are no other suitable alternatives. ${ }^{36}$ The use of nitrofurantoin in pregnancy shows no increased risk for cardiovascular malformations, oral cleft, or craniosynostosis. ${ }^{2,37}$

Chloramphenicol, tetracyclines, and cotrimoxazole should be avoided in pregnancy. ${ }^{2,34}$ However, during early pregnancy chloramphenicol treatment presents little, if any, teratogenic risk to the foetus in humans. ${ }^{2,38}$

The exposure in utero to clindamycin, doxycycline, quinolones, and macrolides are linked to organ-specific malformations whereas exposure to amoxicillin, cephalosporins, and nitrofurantoin are not associated with major congenital malformations. ${ }^{2,38}$ Beta-lactams, vancomycin, nitrofurantoin, and clindamycin are generally considered safe and effective in pregnancy whereas fluoroquinolones and tetracyclines should generally be avoided in pregnancy. 2,39,40

There was poor documentation of repeat cultures, as well as the use of prophylactic urinary antimicrobials to prevent recurrent infections, particularly in those patients with acute 
pyelonephritis. Only 50\% of cases of pyelonephritis had repeat cultures either during admission or at follow up. There was a $0.45 \%$ incidence of pyelonephritis in this study, which is similar to reported ranges from $0.5 \%$ to $2.0 \%$ in the literature. ${ }^{29}$ Urinary tract infections recur in approximately $4 \%-5 \%$ of pregnancies in patients with structural abnormalities of renal system and a single, postcoital dose or daily suppression with cephalexin or nitrofurantoin is an effective preventive therapy. ${ }^{41}$

Some studies demonstrated a relationship between UTI in pregnant women and the risk of poor perinatal outcomes. ${ }^{42}$ However, authors like Chen et al. concluded that there were no increased risks of adverse pregnancy outcomes in women, and neonates born to women with UTIs. ${ }^{43}$

\section{Limitations}

The limitations of this study were the small sample size, missing data in the hospital file and microscopy results, symptoms at time of testing especially as symptoms of UTI and pregnancy are similar, the inherent shortcomings of a retrospective study and that it was confined to one hospital. Approximately $14 \%$ of the files were not retrieved, as some were lost. This study did not differentiate between nosocomial and community acquired infections.

Future prospective studies evaluating the impact of UTI in pregnant women in our setting using a larger sample size is recommended to address this limitation. And an improved patient record system will be beneficial to future studies.

\section{Conclusion}

E. coli, Klebsiella, Enterococcus faecalis and coagulase-negative staphylococcus were the most common microorganisms identified in this study. ${ }^{2}$ Empirical therapy with oral nitrofurantoin and cefuroxime or parenteral ceftriaxone are appropriate. Most of these antibiotics are relatively safe to be used in pregnancy and breastfeeding. ${ }^{2}$ The choice of antimicrobial therapy in pregnancy should be determined according to the sensitivity and resistance for foeto-maternal safety. ${ }^{2}$

\section{Acknowledgement}

I would like to thank Prof H.A. Lombaard for his valuable input; Mr. Thomas Papo of the NHLS and the members of the records department at $\mathrm{RMMCH}$ for providing the data results and case files to conduct this study.

\section{Competing interests}

The authors declare that they have no financial or personal relationships that may have inappropriately influenced them in writing this article.

\section{Authors' contributions}

O.O. was the project leader and Z.D. was responsible for experimental and project design and acted as co-supervisor.
A.W. aided in conceptual contribution, proof read the article, corrected project design and acted as co-supervisor.

\section{Funding information}

The authors received no financial support for the research, authorship and/or publication of this article.

\section{Data availability}

The data that support the findings of this study are available from the corresponding author, O.O, upon reasonable request.

\section{Disclaimer}

The views and opinions expressed in this article are those of the authors and do not necessarily reflect the official policy or position of any affiliated agency of the authors, and the publisher.

\section{References}

1. Onoh RC, Umeora OU, Egwuatu VE, et al. Antibiotic sensitivity pattern of uropathogens from pregnant women with urinary tract infection in Abakaliki, uropathogens from pregnant women with urinary tract infection in Abak
Nigeria. Infect Drug Resist. 2013;6:225. https://doi.org/10.2147/IDR.S46002

2. Matalka AA, Al-Husban N, Alkuran $\mathrm{O}$, et al. Spectrum of uropathogens and their susceptibility to antimicrobials in pregnant women: A retrospective analysis of 5-year hospital data. J Int Med Res. 2021;49(5): 1-14. https://doi. org $/ 10.1177 / 03000605211006540$

3. Nicolle LE, Bradley S, Colgan R, et al. Infectious diseases society of America guidelines for the diagnosis and treatment of asymptomatic bacteriuria in adults. Clin Infect Dis. 2005;40(5):643-654. https://doi.org/10.1086/427507

4. Addo VN. Urinary tract infection in pregnancy. In: EY Kwawukume, EE, Emuveyan, editors. Comprehensive obstetrics in the tropics. Dansoman: Asante and Hittscher Printing Press Limited, 2002; p. 261-267.

5. Borchert D, Sheridan L, Papatsoris A, et al. Prevention and treatment of urinary tract infection with probiotics: Review and research perspective. Indian J Urol. 2008;24(2):139-144. https://doi.org/10.4103/0970-1591.40604

6. Cunningham FG, Gant NF, Leveno KJ, et al. Renal and urinary tract disorders. In: A Seils, SR Noujaim, K Daris, editors. Williams Obstetrics. 21st ed. New York, NY: McGraw-Hill Medical Publishing Division, 2001; p. 1251-1272.

7. Mathia E, Thomas RJ, Chandy S, et al. Antimicrobials for the treatment of urinary tract infection in pregnancy: Practice in southern India. Pharmaco Epidemiol Drug Saf. 2004;13(9):645-652. https://doi.org/10.1002/pds.950

8. Johnson EK, Wolf JS. Urinary tract infections in pregnancy [serial online] Medscape 2021 [cited 2021 June 6]. Available from: http://emedicine.medscape. com/article/452604overview

9. Patterson TF, Andriole VT. Bacteriuria in pregnancy. Infect Dis Clin North Am. 1987;1(4):807-822. https://doi.org/10.1016/S0891-5520(20)30151-3

10. Schieve LA, Handler A, Hershow R, et al. Urinary tract infection during pregnancy: Its association with maternal morbidity and perinatal outcome. Am J Public Health. 1994;84(3):405-410. https://doi.org/10.2105/AJPH.84.3.405

11. Nnatu $S$, Essien EE, AkinKugbe $A$, et al. Asymptomatic bacteriuria in pregnant Nigerian patients. Clin Exp Obstet Gynecol. 1989;16:126-128. https://doi. org/10.4103/1119-3077.122856

12. Ezechi OC, Fasubaa OB, Dare FO. Antibiotic sensitivity patterns of microbia isolates from urine of pregnant women with urinary tract infections. Trop J Obstet Gynaecol. 2003;20(2):113-115. https://doi.org/10.4314/tjog.v20i2.14413

13. Abdul IF, Onile BA. Bacterial isolates from urine of women in llorin and their antibiotic susceptibility patterns. Trop J Obstet Gynaecol. 2001;18(2):61-65. https://doi.org/10.4314/tjog.v18i2.14431

14. Arias F. Abnormalities of the urinary system during pregnancy. In: SN Daftary, AG Bhide, editors. Practical guide to high risk pregnancy and delivery: A South Asian perspective. 3rd ed. New Delhi: Elsevier, 2008; p. 489-505.

15. Alausa KO, Montefiore D, Sobayo E. Problems in the diagnosis of urinary tract infections. Niger Med J. 1979;9:107-111.

16. Onyemenem TN, Ekweozor CC. Urinary tract infection in Ibadan: Causative organisms and antimicrobial sensitivity patterns. Afr J Med Sci. 1996;25:165-169.

17. Wasserman S, Boyles T, Mendelson M. Pocket guide to antibiotic prescribing for adults in South Africa. Cape Town: South African Antibiotic Stewardship Programme, 2015; p. 1-60.

18. Leegaard TM, Caugant DA, Frøholm LO, et al. Apparent differences in antimicrobial susceptibility as a consequence of national guidelines. Vol. 6. In: Leegaard TM editor. Clinical microbiology and infection. Norway: Department of Bacteriology, National Institute of Public Health, 2000; p. 290-293. 
19. Bera E, editor. Rahima Moosa mother and child hospital obstetrics statistics. JanDec 2017. Johannesburg

20. Mail \& Guardian. Three hospitals in Gauteng renamed. Johannesburg: SAPA; 2008 Sep 29.

21. Michael LW, Loretta G. Laboratory diagnosis of urinary tract infections in adult patients. Clin Infect Dis. 2004. 38(8):1150-1158. https://doi.org/10.1086/383029

22. Nabil K, Rayne R, Waal FE, et al. Guideline for urine culture and biochemical identification of bacterial urinary pathogens in low-resource settings Octobe 2020. Diagnostics. 2020;10(10):832. https://doi.org/10.3390/diagnostics10100832

23. Harris PA, Taylor R, Thielke R, et al. Research electronic data capture (REDCap)-A metadata-driven methodology and workflow process for providing translationa research informatics support. J Biomed Inform. 2009;42(2):377-381. https://doi. org/10.3390/diagnostics10100832

24. Harris PA, Taylor R, Minor BL, et al. REDCap Consortium, The REDCap consortium Building an international community of software partners, J Biomed Inform. 2019;95:103208. https://doi.org/10.1016/j.jbi.2019.103208

25. StataCorp. Stata statistical software: Release 16. College Station, TX: StataCorp LLC; 2019.

26. Leeper C, Lutzkanin A. Infections during pregnancy. 3rd ed. Prim Care. 2018;45(3):567-586. https://doi.org/10.1016/j.pop.2018.05.013

27. Szweda $H$, Jozwik M. Urinary tract infections during pregnancy - An updated overview. Dev Period Med. 2016;20(4):263-272.

28. Gilstrap LC III, Ramin SM. Urinary tract infections during pregnancy. Obstet Gynecol Clin North Am. 2001;28(3):581-591. https://doi.org/10.1016/S08898545(05)70219-9

29. Kamgang FD, Maise HC, Moodley J. Pregnant women admitted with urinary tract infections to a public sector hospital in South Africa: Are there lessons to learn? S Afr J Epidemiol Infect. 2016;31(3):22-26. https://doi.org/10.4102/sajid.v31i3.82

30. Lewis DA, Gumede LY, Van der Hoven LA, et al. Antimicrobial susceptibility of organisms causing community-acquired urinary tract infections in Gauteng province, South Africa. S Afr Med J. 2013;103(6):377-381. https://doi. org/10.7196/SAMJ.6722

31. Connolly A, Throp Jr JM. Urinary tract infections in pregnancy. Urol Clin North Am. 1999;26:779-787. https://doi.org/10.1016/S0094-0143(05)70218-4
32. Velisha APP, Suleman F, Quantitative evaluation of essential medicines lists: The South African case study. BMC Health Service Res. 2016;16(1):687. https://doi. org/10.1186/s12913-016-1937-x

33. Bera E. South African National essential medicine list. Adult hospital level, medication review process, component. Johannesburg: Obstetrics. 2017.

34. Czeizel AE. The estimation of human teratogenic/fetotoxic risk of exposures to drugs on the basis of Hungarian experience: A critical evaluation of clinical and epidemiological models of human teratology. Expert Opin Drug Saf. 2009;8:283-303. https://doi.org/10.1517/14740330902916459

35. Nyakoe R. Urinary tract infection. EM guidance. Johannesburg: University of the Witwatersrand; 2018.

36. American College of Obstetricians and Gynecologists, 2017. Sulfonamides, nitrofurantoin, and risk of birth defects. Committee Opinion No. 717. Obstet Gynecol. 2017;130(3):e150-e152. https://doi.org/10.1097/AOG.0000000000002300

37. Goldeberg $\mathrm{O}$, Moretti $\mathrm{M}$, Levy $\mathrm{A}$, et al. Exposure to nitrofurantoin during early pregnancy and congenital malformations: A systematic review and meta-analysis. J Obstet Gynaecol Can. 2015;37:150-156. https://doi.org/10.1016/S17012163(15)30337-6

38. Czeizel AE, Rockenbauer M, Sørensen HT, Olsen J. A population-based case-control teratologic study of oral chloramphenicol treatment during pregnancy. European journal of epidemiology. $2000 \mathrm{Apr} ; 16(4): 323-327$.

39. Muanda FT, Sheehy O, Berard A. Use of antibiotics during pregnancy and the risk of major congenital malformations: A population-based cohort study. Br J Clin Pharmacol. 2017;83(11):2557-2571. https://doi.org/10.1111/bcp.13364

40. Bookstaver PB, Bland CM, Griffin B, et al. A review of antibiotic use in pregnancy. Pharmacotherapy. 2015;35(11):1052-1062. https://doi.org/ 10.1002/phar.1649

41. John ED, Michael LL. Urinary tract infections during pregnancy: University of Missouri-Columbia School of Medicine, Columbia, Missouri. Am Fam Physician. 2000;61(3):713-720.

42. Mead PJ, Harris RE. The incidence of group B beta haemolytic streptococcus in antepartum urinary tract infections. Obstet Gynecol. 1978;51(4):412-414 https://doi.org/10.1097/00006250-197804000-00005

43. Chen YK, Chen SF, Li HC, et al. No increased risk of adverse pregnancy outcomes in women with urinary tract infections: A nationwide population-based study. Acta Obstet Gynecol Scand. 2010;89(7):882-888. https://doi.org/10.3109/00016349.2 010.486826 